$\mathbb{P}$ periodica polytechnica

Civil Engineering

$56 / 1$ (2012) 3 -11

doi: 10.3311/pp.ci.2012-1.01

web: http://www.pp.bme.hu/ci

(c) Periodica Polytechnica 2012

RESEARCH ARTICLE

\section{Validation of GOCE time-wise gravity field models using GPS-levelling, gravity, vertical deflections and gravity gradient measurements in Hungary}

Eszter Szúcs

Received 2011-09-19, revised 2012-01-12, accepted 2012-02-12

\begin{abstract}
With the advantage of satellite gravity gradiometry (SGG) high standard global gravity determination could attain in the static part of the gravitational field. This paper presents the validation of the first and second generation GOCE-only models using terrestrial data sets in Hungary. GOCE global geopotential models (GGM) are consistent models with global coverage in sense that GGMs have been compiled utilizing measurements refer to short time period. Besides GOCE-based GGMs satellite only GRACE models were evaluated to assess the improvements by GOCE observations with respect to GRACE in gravity field determination. EGM2008 as the state-of-the-art model and SRTM3 elevation model were applied to provide that measurements involving Hungarian data sets and model derived gravity field functionals have almost the same spectral content. Results with GPS-levelling and gravity data support that there is an improvement in the determination of medium-wavelength parts of the gravitational field with GOCE models. Although vertical deflections characterize the short-wave part of the gravity field, they are also capable to sense the advancement of SGG observations. Our experiences show that torsion balance measurements depict the fine structure of the gravity field, and hence they are not adequate in low-degree GGM validation.
\end{abstract}

\section{Keywords}

GOCE $\cdot$ EGM2008 - SRTM3 - GPS-levelling $\cdot$ gravity $\cdot$ vertical deflections $\cdot$ gravity gradients

\section{Acknowledgement}

The careful and thoughtful reviews of the reviewers (J. Benedek and L. Földváry) are greatly appreciated as well as the use of GRAVSOFT software by C.C. Tscherning and R. Forsberg.

\section{Eszter Szücs}

Geodetic and Geophysical Institute, Research Centre for Astronomy and Earth Sciences, Hungarian Academy of Sciences, H-9400 Sopron, Csatkai u. 6-8, Hungary

e-mail: szucs_e@ggki.hu

\section{Introduction}

CHAMP, GRACE and GOCE dedicated satellite missions [22] contribute to global determination of the longwavelength parts of the gravity field (e.g. [5, 14, 15]). ESA's Gravity field and steady-state Ocean Circulation Explorer is the first dedicated satellite mission that employs gravity gradiometry to determine and refine the long- and especially the medium-wavelength components of the Earth's gravitational field. The satellite has launched on 17 March 2009, and since there have been released first and second generation gravity field solutions, based on 2 and 6 months data sets respectively (third generation GOCE models have been released since the submission of the manuscript). A remarkable advantage of the recently released GOCE GGMs is that they provide determination of the Earth's gravitational field with high accuracy since the GOCE data cover homogeneously the entire Earth - without the unobserved polar caps of $6.5^{\circ}$ spherical radius- and refer to a short time span. Via International Centre of Global Earth Models (www.icgem.com) three different geopotential model solutions are available depending on the incorporated data sets and computation method. The direct (DIR) and space-wise (SPW) GOCE global geopotential models starts with an a-priori gravity field model as an a-priori information while the time-wise (TIM) GGMs based on solely GOCE data. The DIR solution apply a-priori gravity field information as a background reference model, namely EIGEN5C and ITG-Grace2010S geopotential models for the first and second generation DIR solutions respectively. The first released SPW model makes use of the GOCE quick-look model and EGM2008 for modelling degree variances as prior information; while EIGEN5C was used for signal covariance modelling for second released SPW model. The TIM solutions [18, 19] comprise GOCE data exclusively; where the low frequency components of the gravitational field are derived from high-low satellite-to-satellite tracking method, while satellite gravity gradiometry serves as a source of determining the medium-wavelength features of the gravity field.

Since GOCE TIM models are independent of any gravity field information and do not employ any terrestrial data set they provide a reasonable and unbiased comparison with the available 
ground-truth measurements.

\section{Computational procedure}

In the evaluation of GOCE and GRACE models the different spectral content of the GGMs and terrestrial data has to take into account. Comparing gravity field functionals computed from GGM via spherical harmonic synthesis with terrestrial data sets are not adequate since geopotential model has limited spectral content due to the truncation of the model while terrestrial observations contain the full spectral information. The error, which occurs by truncating the model to a specific degree is the omission error. The maximum half wavelength that is resolved by the GGM is $180^{\circ} / \mathrm{n}$, where $n$ is the maximum degree of the truncated model. Hence gravity field characteristics smaller than the spatial resolution of the model are not represented in the computed gravity functionals. Although the highest power of the gravitational potential can be found at the lowest spherical harmonics, the omission error for e.g. height anomaly is approximately $0.3 \mathrm{~m}$ truncating the GGM at degree 250 (the maximum degree of the second generation GOCE timewise model) according to the Tscherning-Rapp degree variance model. The spectral content of low degree Earth's gravitational models could be augmented utilizing the ultra high resolution EGM2008 model [21]. EGM2008 geopotential model is complete to spherical harmonic degree and order 2,160 and provides some additional coefficients up to degree 2,190. The spatial resolution of EGM2008 is 5 arc min (corresponds to approximately $9 \mathrm{~km}$ depending on latitude), therefore some omission error remains after extension the low degree GGM with the EGM2008 spherical harmonic coefficients. Nevertheless, the remaining omission error could be approximated by Residual Terrain Modelling (RTM), since the topographic masses are the dominant sources of the high frequency parts of the gravitational field of the Earth. Approximation of the omission error in this way is widespread applied in low degree geopotential validation [6,-8, 10]. In our investigations we used the spectral enhancement method (SEM) developed by Hirt et al. [10] to evaluate the first generation GOCE gravity field models using terrestrial data sets. For detailed description about the SEM the reader is referred to [10]. Briefly the principle of the SEM and GGM validation is the following:

1 The GGM under evaluation is truncated to a specific spherical harmonic degree $n_{t r}$, where the initial value for $n_{t r}$ is 2 , which is incremented by 1 until it reaches the maximum spherical harmonic degree of the GGM.

2 EGM2008 is applied to reconstruct the spectral content of the gravitational field from degree $n_{t r}+1$ to degree 2160 .

3 The desired gravity field functional is computed via spherical harmonic synthesis.

4 The remaining gravity field signal (above EGM2008 spectral content) is approximated by forward modelling method com- puting RTM corrections provided by SRTM3 [13] surface elevation set. Closed formula of the gravitational attraction of the prism exists for determining RTM gravity field quantities [4, 16]. RTM was constructed as the difference between the detailed SRTM3 surface model (having spatial resolution of $3 \mathrm{arcsec}$ ) and a smooth reference surface. The reference elevation set was produced from the SRTM3 model applying a Gaussian low-pass filter, the resolution of the filtered grid was adapted to the maximum degree of the EGM2008 model.

5 The GGM (low degree GGM augmented with EGM2008 coefficients) and RTM derived gravity field functional is subtracted from the available measurements and standard deviation of the differences was computed. Varying truncation degree $n_{t r}$ from 2 to $n_{\max }$ (the maximum degree of the GGM under investigation) stepwise and computing standard deviations of the differences for each truncation degree allows to estimates the spectral content of the GGM under evaluation. Since steep slope in the standard deviation (STD) curve (rapidly increasing dispersion values) indicates that the GGM derived gravity field quantity does not fit to the terrestrial measurement and imply spherical harmonic degree of signal loss for the geopotential model.

The above computational scheme has been applied to the available GPS-levelling, free-air gravity anomaly and vertical deflections for Hungary. Detailed description of the data sets is given in Section 3. There exist more than 100,000 surface gravity gradient measurements in Hungary over an area of about $45,000 \mathrm{~km}^{2}$. These measurements are basic tools to study the short- and very short-wavelength part of the gravitational field. Hence they supply a great opportunity to validate the high degree EGM2008 model and investigate the performance of the new generation SGG based geopotential models.

We acknowledge that other methods exist to assess the performance of low-degree GGMs complete to different spherical harmonic degree, e.g. compare local or regional grids of various functionals of the disturbing potential computed to identical spherical harmonic degrees [12], but these topics are beyond the scope of this present study.

\section{Date sets}

\subsection{Global geopotential models}

In our investigations we used the first (GOCE TW1) and second (GOCE TW2) generation time-wise GOCE gravity field models, which have a spectral resolution to spherical harmonic degree and order 224 and 250 respectively. This maximum degree of expansion corresponds to approximately $90 \mathrm{~km}$ and 80 $\mathrm{km}$ spatial resolution for the GOCE TW1 and GOCE TW2 models. The target resolution of the GOCE GGMs is the spectral band from degree 50 to degree 200 [7], so in the determination of the low- and medium-wavelength components of the gravitational field some improvement would be expected. Since GOCE time-wise GGMs are based on measurements collected 
during a short time period and provide a consistent spherical harmonic set, they can apply to identify long- wavelength patterns e. g. slopes in differences between GPS-levelling and GGM quasigeoid heights. According to the spatial resolution of the GOCE models, gravity field features smaller than $\sim 100 \mathrm{~km}$ are not involved in the computed quantities, hence GOCE GGMs could be applied in regional geoid investigations. To estimate the quality of the GOCE time-wise models two GRACE-based models were applied in the computation procedure described in Section 2. These GGMs are the ITG-GRACE03S [14] and ITG-GRACE2010S [15] models based on GRACE intersatellite ranging and hl-SST observations. The computed GGMs incorporate about 5 and 7 years GRACE data sets respectively. These models are complete to spherical harmonic degree and order 180. ITG-GRACE03S is the basis of the low-wavelength part of EGM2008. EGM2008 is considered as a state-of-the-art GGM [17]. It is a combined Earth's gravitational model utilizing satellite, 5 arc min surface terrestrial gravity, elevation and altimetry data. This model was applied for approximating the omission error as described in Section 2 and was used as a reference for evaluating the other models.

Table 1 gives an overview of the used GGMs. In our study we used the zero-tide version of the models, i.e. the direct effect of the gravitational attraction of Sun and Moon are removed, while the indirect effect component related to the elastic deformation of the Earth is retained. We chose this tide-system since it is well-defined, converting spherical harmonics from tide-free to zero-tide system is given by [11]. The conversion concerns only the $C_{20}$ coefficient. In GGM evaluation the GRS80 normal field was used.

Tab. 1. Overview of the investigated GGMs

\begin{tabular}{ccc}
\hline GGM name & $\begin{array}{c}\text { Model resolution } \\
\text { (maximum } \\
\text { degree/order) }\end{array}$ & $\begin{array}{c}\text { Description, data } \\
\text { source }\end{array}$ \\
\hline EGM2008 & 2190 & $\begin{array}{c}\text { GRACE, terrestrial } \\
\text { gravity, elevation and } \\
\text { altimetry data }\end{array}$ \\
\hline GOCE TW1 & 224 & $\begin{array}{c}\text { GOCE time-wise } \\
\text { approach, } \\
\text { st }\end{array}$ \\
generation model \\
\hline GOCE TW2 & 250 & $\begin{array}{c}\text { GOCE time-wise } \\
\text { approach, } \\
2^{\text {nd }} \text { generation } \\
\text { solution }\end{array}$ \\
\hline ITG-GRACE03S & 180 & $\begin{array}{c}\text { Based on GRACE } \\
\text { K-band intersatellite } \\
\text { ranging and SST. } \\
\text { Form the base of } \\
\text { EGM2008. }\end{array}$ \\
\hline ITG-GRACE2010S & 180 & $\begin{array}{c}\text { Based on GRACE } \\
\text { K-band intersatellite } \\
\text { ranging and SST. }\end{array}$ \\
\hline
\end{tabular}

Geopotential models are given as a series of spherical har- monic coefficients with the associated errors for various degrees hence the models can be tested in the spectral domain which means an internal validation. Since the coefficients of the GGMs are given with respect different defining $G M$ - product of gravitational constant and mass of the Earth - and $R$ - reference radius - parameters the coefficients have to be rescaled in order to perform an adequate comparison of the computed degree specific quantities [26]. A scale factor was calculated for each spherical harmonic coefficient of the investigated GOCE and GRACE model based on the reference $G M$ and $R$ values of EGM2008

$$
f=\frac{G M^{G G M}}{G M^{E G M 2008}}\left(\frac{R^{G G M}}{R^{E G M 2008}}\right)^{n},
$$

where $n$ is the degree of the spherical harmonic coefficient. Fig. 1 displays the computed scaled geoid signal and error degree variances. From Fig. 1 it can be seen that GGMs based on exclusively GRACE observations have the same power as EGM2008 about up to degree 150 and degree 160 for the ITGGRACE03S and for the ITG-GRACE2010S respectively. Regarding to the GOCE time-wise models, GOCE TW1 retains full power up to degree 190, while GOCE TW2 has the same power as EGM2008 to about degree 200 approximately. Fig. 1 displays that constrains towards to zero have been applied to high degree harmonic coefficients of GOCE time-wise gravity field solutions to improve signal-to-noise ratio. Error degree variance curves show that GOCE time-wise models at low degrees are not competitive with GRACE based models which is attributable to the fact that GOCE GGMs were compiled utilizing only 2 and 6 months long measurements. From the cumulative error degree variance curves (Fig. 2) it can be concluded that ITG-GRACE models offer $\mathrm{cm}$ accuracy geoid up to degree 119 and 135 for ITG-GRACE03S and ITG-GRACE2010S respectively. GOCE time-wise solutions do not perform as well as ITG models regarding to the cumulative geoid errors, nevertheless GOCE error curves are not as steep as GRACE-based models, which can be regarded as an improvement. The 6-month GOCE TW2 solution shows progress in geoid error compared to 2-month time-wise gravity field solution. Probably wider time span based GOCE models would improve the accuracy of geoid determination since new GOCE models are based only few month long data sets while GRACE models incorporating measurements collected through several years. Hence our study can be regarded as a preliminary result, but we think that it is worth to estimate what improvements could be derived from the new SGG based gravity field models.

\subsection{Terrestrial data sets}

The horizontally extension of the data sets (approximately $310 \mathrm{~km} \times 500 \mathrm{~km}$ ) compared with the spatial resolution of GOCE GGMs is suitable to make the analysis spectrally representative. The available data sets for GGM evaluation were freeair gravity anomalies at 1243 points, GPS/levelling observations of the Hungarian GPS Network (OGPSH) at 330 points [28] and 


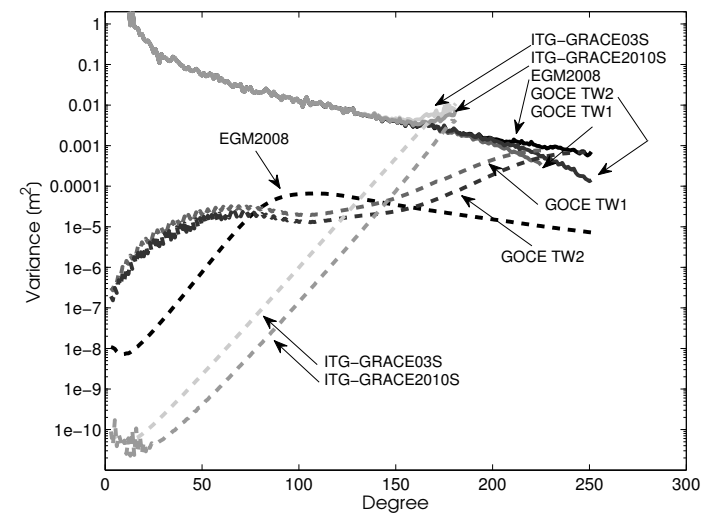

Fig. 1. Geoid signal (solid line) and error (dashed line) degree variances for the investigated models

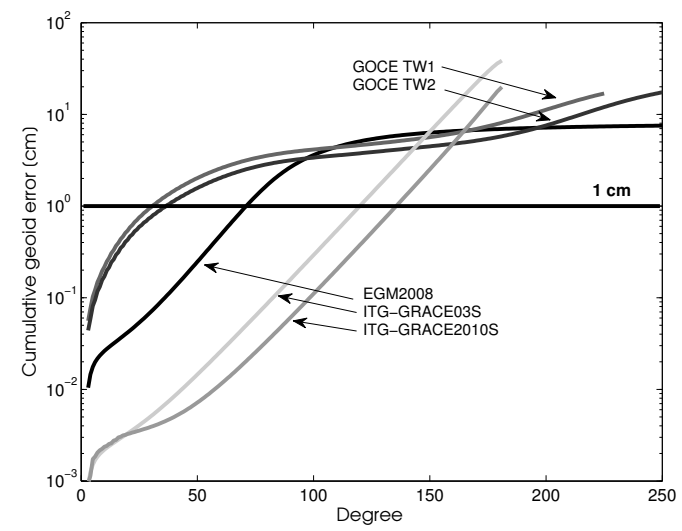

Fig. 2. Cummulative geoid error of the various GGMs

138 astrogeodetic deflections of the vertical, both meridian and prime vertical components. Distribution of data is near uniform in the whole country, the average point distance is about $9 \mathrm{~km}$, $17 \mathrm{~km}$ and $27 \mathrm{~km}$ for the gravity, GPS-levelling and vertical deflections data set. Torsion balance developed by Eötvös, was the first dedicated gravity gradiometer. For detailed description of the torsion balance, measurement methods and interpretation of observation the reader is referred to Hungarian geodetic literature (e.g. [2, 23, 24]). Torsion balance can measure some components of the gravity gradient tensor. Gravity gradients contain local gravity field information, and could apply in high-resolution gravity field determination. Since removerestore method is widely applied in gravity field modelling to make observations statistically homogeneous, the investigation of the performance of the high resolution EGM2008 model in terms of gravity gradient comparison is a new opportunity to us. In our study we used the horizontal gradients of the vertical component of the gravity vector or simply called horizontal gravity gradients, which measure the non-paralellism of potential surfaces. Horizontal gradients were available at 24950 points; most of the torsion balance sites are located on low-elevated terrain (RMS of station heights is $130 \mathrm{~m}$ ), few stations are situated in mountain valleys.

\section{Evaluation results}

Comparing GGM derived gravity field functional with measurements allows the external validation of the models. Utilizing geoid and gravity information the medium- and short- wavelengths part (degree 50-200) of the gravity field can be tested [8] which equals to the target spectral window of GOCE models.

In the next subsections the evaluation results of the different data sets using SEM is discussed. Tab. 2 reports the statistics of the differences between observations and EGM2008 (to degree 2160) and EGM2008 augmented by RTM functionals of the gravity field. These values were used as benchmark to quantify the quality of the investigated GGMs. In case of height anomaly, gravity anomaly and vertical deflections the low standard deviation values indicate the good accordance between terrestrial measurements and EGM2008 and RTM gravity field functionals. Results obtained for horizontal gradients are discussed in Sec. 4.4. Omission error was approximated for GPS-levelling, gravity and vertical deflections beyond degree 2160 with RTM gravity field quantity. Torsion balance data set is supplemented with topographic correction data which accounts for the effect of the nearby and remote terrain relief. Since gravity gradient measurements are very sensitive to local masses these topographic effects were applied instead of computing RTM gravity gradients. We chose this way of handling the spectral content of the gravity field for gravity gradients beyond degree 2160 because there is no freely available high resolution terrain model for Hungary. SRTM3 is a surface model and the difference between surface and terrain model could modify the computed RTM gradients depending on the height of the vegetation [20]. Furthermore, torsion balance stations are situated on low-lying terrain part of Hungary and investigations have shown [9] that in lesselevated areas RTM gravitational functionals are more affected by SRTM model errors. Additionally our investigations with gravity gradients should be regarded as a coarse assessment of what improvements of SGG measurements may make up in the determination of the main features of gravity gradients.

In the SEM procedure for all combination degree $\left(n_{t r}\right)$ the mean value of the differences was computed, and then was subtracted from the result in order to take into account the inconsistencies in reference system definition for all data sets. Since EGM2008 model after truncation was always enhanced with EGM2008 coefficients, the computed standard deviation of the differences is equal at all truncation degrees.

\subsection{Results using GPS-levelling data set}

Fig. 3 shows the standard deviation of the height anomaly differences for all gravity field models which were involved in this investigation as a function of spherical harmonic band from $n=$ 2 to truncation degree $\left(n_{t r}\right)$. From the steepness of the slopes of STD curves the spherical harmonic degree of signal loss can be identified, since increasing dispersion of height anomaly differences implies the degree of truncation where the GGM fits no longer to terrestrial data set. All GGMs show a comparable 
Tab. 2. Statistics of the original as well as EGM2008 (d/o 2160) and EGM2008/RTM reduced observations. In case of gravity gradients topographic corrections (TC) was applied instead of computing RTM gravity gradients

\begin{tabular}{|c|c|c|c|c|c|}
\hline data type & Difference & mean & std. & $\min$. & $\max$. \\
\hline GPS-levelling & observation & 43.018 & \pm 1.718 & 38.851 & 46.286 \\
\hline \multirow[t]{2}{*}[\mathrm{m}]{} & obs. - EGM2008 & 0.104 & \pm 0.062 & -0.112 & 0.425 \\
\hline & obs. - EGM2008/RTM & 0.109 & \pm 0.062 & -0.109 & 0.430 \\
\hline grav. anom. & observation & 32.148 & \pm 17.199 & 4.050 & 117.300 \\
\hline \multirow[t]{2}{*}{ [mGal] } & obs. - EGM2008 & 11.905 & \pm 5.265 & -18.546 & 46.949 \\
\hline & obs. - EGM2008/RTM & 11.497 & \pm 3.435 & -7.273 & 25.119 \\
\hline defl. N-S & observation & 0.55 & \pm 2.47 & -7.33 & 6.35 \\
\hline \multirow[t]{2}{*}{ [arcsec] } & obs. - EGM2008 & -0.16 & \pm 0.85 & -3.14 & 2.58 \\
\hline & obs. - EGM2008/RTM & -0.22 & \pm 0.61 & -2.27 & 1.41 \\
\hline defl. W-E & observation & 2.85 & \pm 2.17 & -3.09 & 8.16 \\
\hline \multirow[t]{2}{*}{ [arcsec] } & obs. - EGM2008 & 0.00 & \pm 0.83 & -3.14 & 2.95 \\
\hline & obs. - EGM2008/RTM & -0.04 & \pm 0.61 & -2.33 & 1.95 \\
\hline Tzx & observation & -0.9 & \pm 14.5 & -203 & 126.8 \\
\hline \multirow[t]{2}{*}{ [E] } & obs. - EGM2008 & -0.5 & \pm 14.5 & -184.1 & 133.3 \\
\hline & obs. - EGM2008/TC & -0.2 & \pm 12.9 & -138.0 & 148.9 \\
\hline Tzy & observation & 1.0 & \pm 14.5 & -151.6 & 134.12 \\
\hline \multirow[t]{2}{*}[E]{} & obs. - EGM2008 & 0.9 & \pm 14.4 & -167.5 & 187.8 \\
\hline & obs. - EGM2008/TC & 0.6 & \pm 12.9 & -189.8 & 246.7 \\
\hline
\end{tabular}

performance at low degrees, about up to degree 150. For the GRACE-based satellite only models the standard deviation of the height anomaly differences start to increase at degree $\sim 150$ and degree $\sim 160$ for ITG-GRACE03S and ITG-GRACE2010S respectively. The 2-month GOCE time-wise model experience signal loss at degree 185 while 6-moth GOCE model performs much better, it exhibits more signal content, namely to degree $\sim 210$.

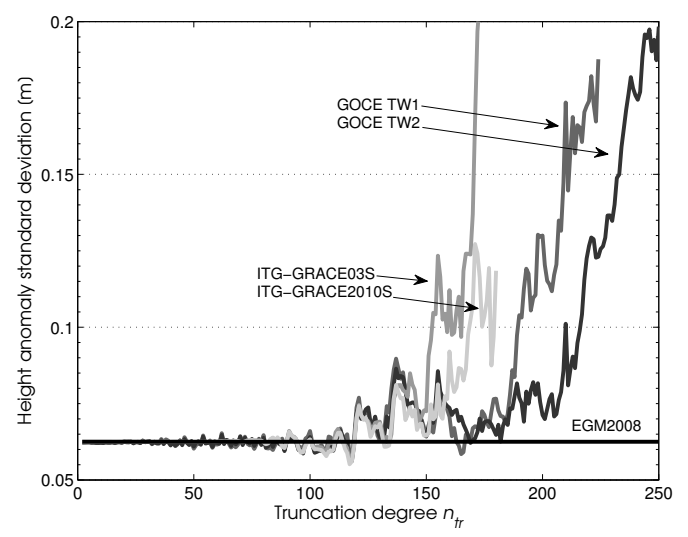

Fig. 3. Standard deviation of height anomaly differences as a function of truncation degree

Applying GPS-levelling quasigeoid height for gravity field validation is commonly used method in geodesy [17]; although separating levelling and GPS errors can be problematic, moreover the quality of GPS-levelling measurements sometimes can be ambiguous. GGMs can be applied to identify inconsistencies in GPS-levelling data sets [6], especially when long-wavelength patterns arise in quasigeoid height differences.

There are some issues with the consistency of the Hungarian GPS-levelling data set [24]. Recent studies on the re-levelling of northeast polygons of the national vertical network revealed surface subsidence in the east part of the country in magnitude approximately $10 \mathrm{~cm}$ during a 30 years time span [1]. This part of the country is situated on unconsolidated sediments and compaction of the sediments probably causes surface subsidence. Since the southeast part of the country lies on area completely covered by sediments further subsidence is expected. GOCE time-wise GGMs are based on solely GOCE observations hence supply homogeneous gravity field information for a definite time epoch. Resolution of GOCE GGMs (Section 3.1) allows us to manifest gravity field characteristics having wavelength more than $100 \mathrm{~km}$. The area with suspected subsidence has an extension of about longitude $20^{\circ}-21^{\circ}$ and latitude $46^{\circ}-47^{\circ}$. Dividing the country into 4 region at longitude $19.5^{\circ}$ and latitude $47^{\circ}$ and applying the SEM method for each region; we can determine the truncation degree from which the GGM derived height anomaly is not consistent with the terrestrial data set. For the north- and southwest regions it can be concluded that there is a clear signal improvement in determining the gravity field from approximately degree 160 to degree 210 for the GOCE TW2 model. The 2-month GOCE model slightly performs worse, signal loss can be detected at about degree 160. GRACE satellite-only models manifest signal loss at degree 145 and degree 160 in the northwest region for ITG-GRACE03S and ITG-GRACE2010S respectively, while they show almost same performance in southwest region of the country. As far as the east part of the country is concerned the STD of the height anomaly differences varies with different amplitudes, a clear steep gradient in STD curves cannot be exhibited (Fig. 4 right side). STD of the differences in the northeast part of Hungary is increasing from degree 135 and oscillating with enlarging amplitudes. As far as the southeast region is concerned none of the models fits to the GPS-levelling data set beyond spherical harmonic degrees 135, a steep gradient in STD curves cannot be detected. Although the extension of the area suspected with subsidence is on the limit of the GOCE 

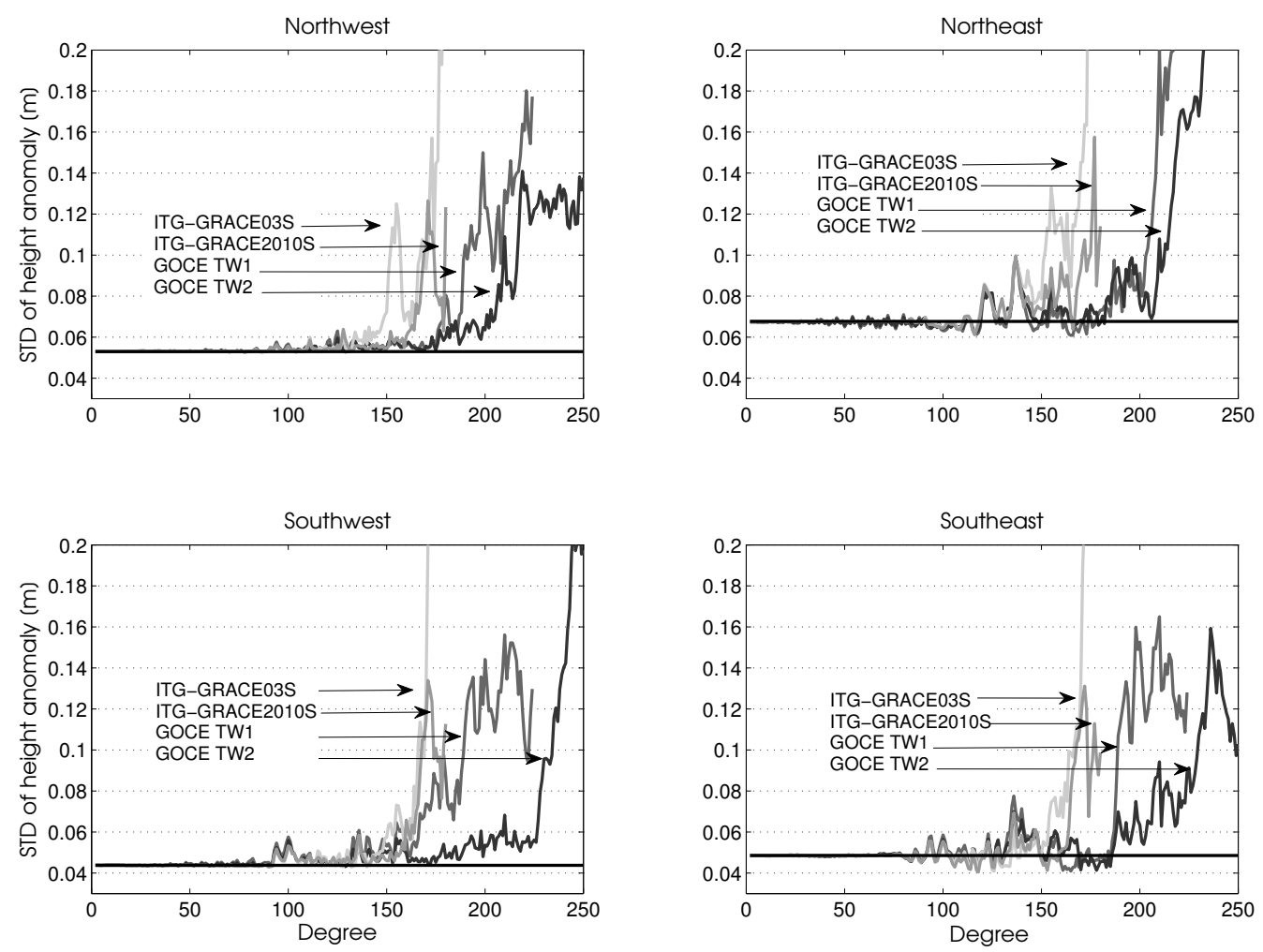

Fig. 4. Standard deviation of quasigeoid height differences for various truncation degrees in 4 region. Black straight line denotes EGM2008 only solution

models' resolution, it can be concluded from the above discussion that inconsistencies in quasigeoid height differences might justify utilizing the recently released GOCE GGMs.

\subsection{Results using free-air gravity anomalies}

The SEM evaluation results for gravity anomalies are displayed in Fig. 5. Up to degree 150 the satellite-only models show similar performance as the combined EGM2008 model. STD values for both GRACE-only models strongly increase from about degree 160. STD of gravity anomaly differences starts to rise for GOCE-based models roughly 30-40 spherical harmonic degrees later. It indicates, that there is a clear improvement in determination of the gravity field in spectral band from 160 to 180 and for GOCE TIM1 and spectral range between 160 to 210 for GOCE TIM2 respectively, compared to GRACE satellite-only models. The 6-month GOCE model shows significant improvement to the previous first generation GOCE TIM1 model.

\subsection{GGM validation utilizing vertical deflection}

Fig. 6 shows the STD of vertical deflection differences, i.e. measured deflection minus GGM derived and RTM deflections. The SEM method was used to ensure that measurements and computed quantities have approximately the same spectral content. Since vertical deflections are more sensitive to local mass distribution and characterize the short wave part of the gravity field of the Earth, it is interesting to analyse that the new

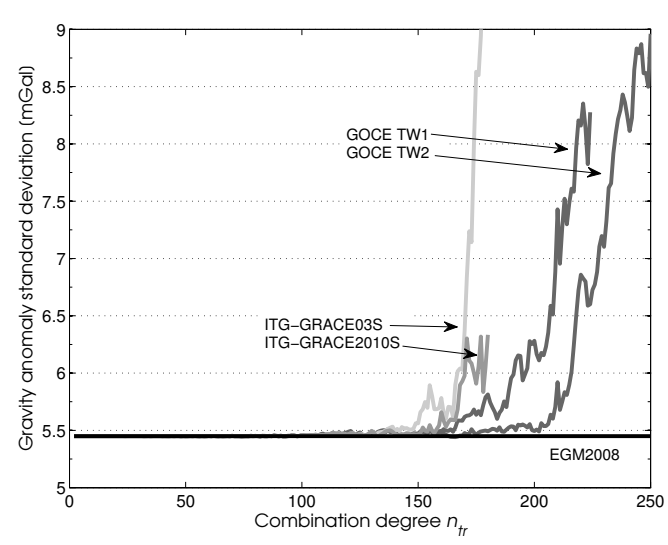

Fig. 5. STD of gravity anomaly differences as a function of truncation degree

SGG-based GGMs are capable to derive reasonable vertical deflections for high truncation degree. Analysing the STD curves of the differences it can be noticed that GOCE models, especially the GOCE TW2, exhibit a significant improvement of the knowledge of the gravity field in spectral band $\sim 165$ - 210 with respect to the GRACE-only models. ITG-GRACE03S and ITGGRACE2010S are very similar in performance. The models experience signal loss about spherical harmonic degree 165 and 170 for the North-South $(\xi)$ and for the East-West $(\eta)$ vertical deflections components respectively. GOCE models show similar result for both $\xi$ and $\eta$ components. STD values for the GOCE TW1 start strongly increase at degree $\sim 190$, hence it 

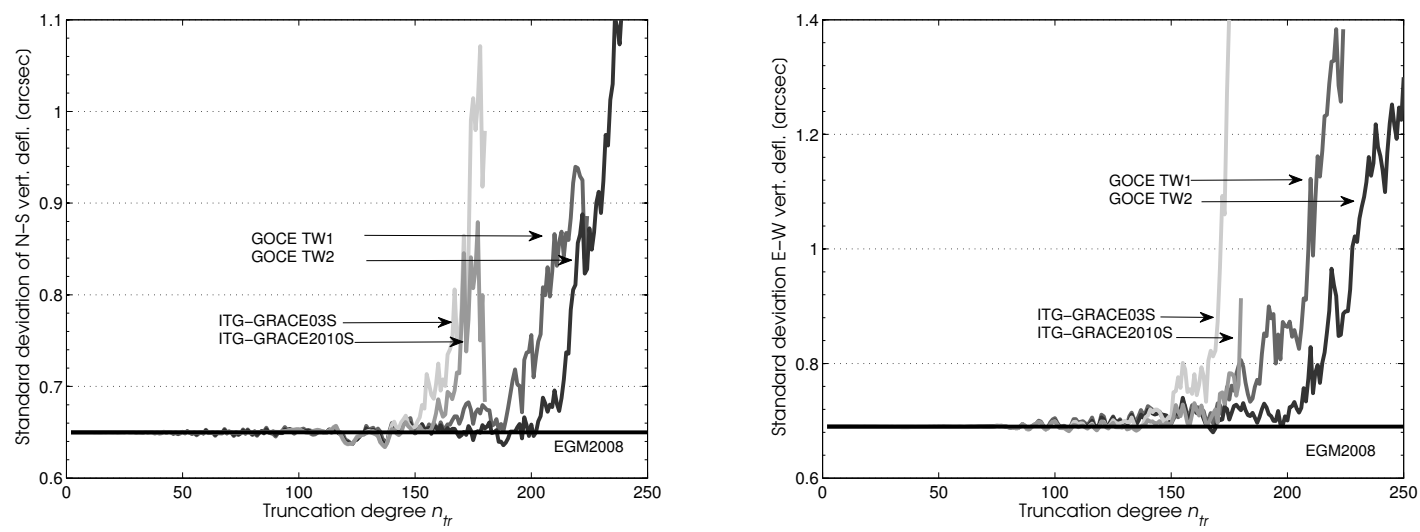

Fig. 6. Standard deviation of vertical deflections differences of varying truncation degrees. Left: North-South, right: West-East component

provides some advancement in gravity field knowledge up to this spherical harmonic degree. Second generation GOCE TIM model experiences signal loss near degree 210, which suggests superior performance of the GOCE TIM2 model compared to other satellite-only models under evaluation [27]. From these result one can conclude that terrestrial vertical deflections are capable to indicate the improvements of high degree gravity signal determination based on SGG measurements.

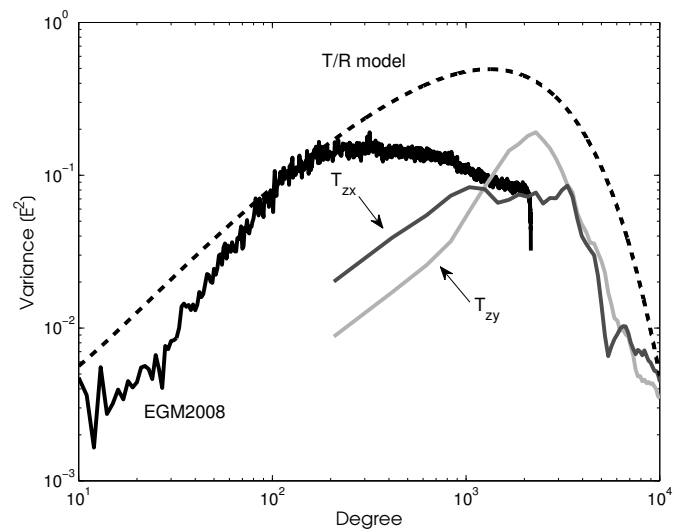

Fig. 7. Degree variances for horizontal gravity gradients from spherical harmonic model EGM2008 (solid line) and computed from Tscherning-Rapp model (dashed line), as well as determined from torsion balance measurements

\subsection{GGM validation using the horizontal gravity gradients}

Surface gravity gradients are rather sensitive to local mass anomalies and characterize the very short-wavelength part of the Earth's gravity field. Since the ultra high degree EGM2008 geopotential model is available it is informative to assess what improvements may make this model in high frequency geoid determination. Statistics of the observed and the EGM2008 reduced gravity gradients are given in Tab. 2. Decreasing of the standard deviation of the gravity gradients after reduction with the EGM2008 model is negligible. Further reduction with topographic effect tapers the STD values slightly, about with 10 $\%$. Since the accuracy of torsion balance measurements of gravity gradients is approximately $\pm 1 \mathrm{E}$, therefore decreasing of the standard deviation during reduction procedure is not significant.
From the figures of the statistics it can be concluded, that the high resolution EGM2008 model is not capable to describe the high frequency part of the gravitational field, Fig. 7 might represents the reasons of it. Fig. 7 shows the degree variances for horizontal gravity gradients determined from the spherical harmonic coefficients of EGM2008 model and from measurements. Degree variances of measured gradients were computed by FFT. Detailed description of the computational method can be found in [3] therefore not repeated here. Signal power of measured and topographic effect reduced gradients was computed for a selected test area having size of $70 \mathrm{~km} \times 145 \mathrm{~km}$ densely covered by torsion balance sites without data gaps to avoid distortion of the estimated spectra. The extension of the selected area determines the lowest degrees of degree variance computation, while the sampling interval $(1.5 \mathrm{~km})$ limits the upper bound of the recovered degree variances. As Fig. 7 illustrates the horizontal gradients have their maximal signal power mainly beyond the spectra covered by the EGM2008 model. Fig. 7 also shows degree variances derived from Tscherning-Rapp model [25]. Although this model is based on free-air anomalies it is suitable to characterize the spectral behaviour of gravity gradients.

The SEM evaluation results for horizontal gravity gradients are displayed on Fig. 8 It is clearly visible that GOCE GGMs show improvement in gravity field knowledge compared to the GRACE only models. In case of the prime vertical component of horizontal gradient $\left(T_{z x}\right)$ GOCE models are very similar in performance, GOCE time-wise gravity field solutions advance the determination of the gravity field in spectral band $\sim$ 160-200. Analysing the STD curves of the differences for the meridian component $\left(T_{z y}\right)$ the GOCE TIM1 only shows a slight improvement compared to ITG-GRACE models, while the 6month GOCE model enhances gravity field knowledge between about spherical harmonic degree 170 to 200 .

\section{Summary and conclusions}

The purpose of this paper is to assess the performance of the Earth's gravity field models based on satellite gradiometry. We have evaluated the 2- and 6-month GOCE time-wise GGMs as 


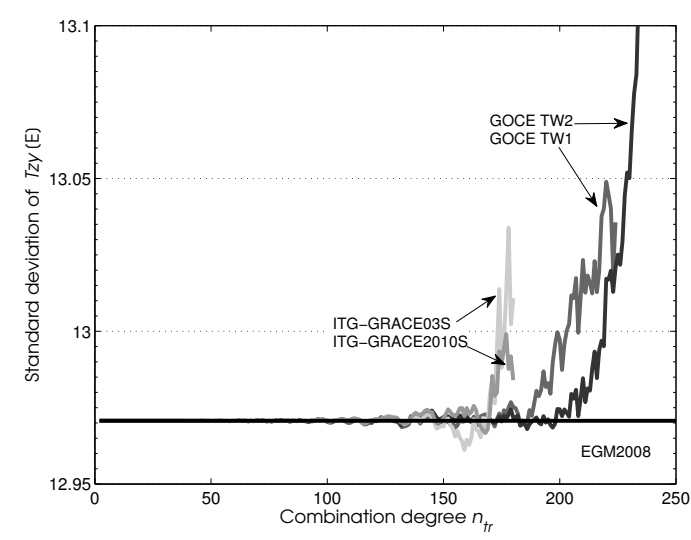

Fig. 8. STD curves of the differences of measured and computed horizontal gradients as a function of truncation degree. Left: meridian component, right: prime vertical component

well as two GRACE only solutions, ITG-GRACE03S and ITGGRACE2010S. Since satellite only models have limited spectral content of the gravitational field, the EGM2008 model was used with residual terrain modelling to provide spectral consistency between measurements and GGM derived gravity field functionals.

The internal accuracy of the investigated GGMs were determined via error spectrum estimation from spherical harmonic coefficients and associated errors. GRACE-based models provide more accurate geoid determination in terms of geoid error degree variances for the long wavelengths yet, probably longer time span GOCE data would improve the accuracy of geoid determination.

Comparison against terrestrial data sets using SEM revealed that the new SGG-based models exhibit improvements in the determination of the static part of the Earth's gravitational field with respect to GRACE-only models. Results obtained from quasigeoid height differences supports that the GOCE time-wise models, especially the 6-month model refine our knowledge of the medium wave part of the gravity field. Comparison of gravity anomalies computed from GGMs with measured ones has shown similar clear indications in spectral band $\sim 160-200$ degree. Even vertical deflections, which are more sensitive to local mass distributions, were capable to sense the improvements of the GOCE models. The second generation GOCE time-wise model has a superior performance compared to other models under evaluation, it experiences signal loss near degree 210. Analysis of horizontal gravity gradients differences showed that torsion balance measurements contain the very high frequency part of the gravitational field.

Our investigations confirmed that GOCE data provide significant new information about the medium wavelength components of the Earth's static gravitational field. Although GOCE time-wise GGMs have not reached their nominal spectral resolution yet, i.e. spherical harmonic degree and order 224 and 250; we can expect that further GOCE data series would improve the models.

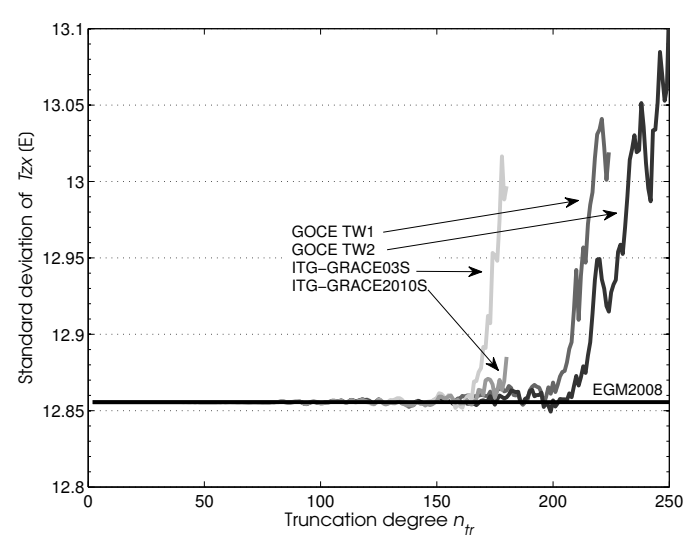

\section{References}

1 Busics G, Preliminary results of the re-measurement of Hungarian National Vertical Network in the first 3 polygons, Geomatikai Közlemények, 13(2), (2010), 141-148. (in Hungarian with English abstract).

2 Csapó G, Laky S, Égető C, Ultmann Z, Tóth G, Völgyesi L, Test measurements by Eötvös torsion balance and gravimeters, Periodica Polytechnica Civil Engineering, 53(2), (2009), 75-80, DOI 10.3311/pp.ci.2009-2.03.

3 Flury $\mathbf{J}$, Short-wavelength spectral properties of the gravity field from a range of regional data sets, Journal of Geodesy, 79, (2006), 624-640.

4 Forsberg R, Terrain effects in geoid computations, International School for the Determination and Use of the Geoid. Lecture Notes, International Geoid School, Milan, Italy, 1994.

5 Földváry L, Bokor Z, Determination of a CHAMP gravity model based on the Newtonian equation of motion, Periodica Polytechnica Civil Engineering, 54(2), (2010), 155-1 61, DOI 10.3311/pp.ci.2010-2.11.

6 Gruber T, Evaluation of the EGM2008 gravity field by means of GPSlevelling and sea surface topography solutions, External quality evaluation reports of EGM08, Newton's Bulletin, 4, (2009), 3-17. Bureau Gravimétrique International (BGI) / International Geoid Service (IGeS).

7 Gruber T, Visser P, Ackermann C, Validation of GOCE gravity field models by means of orbit residuals and geoid comparisons, Journal of Geodesy, 85(11), (2011), 845-860, DOI 10.1007/s00190-011-0486-7.

8 Gruber T, Validation concepts for gravity field models from new satellite missions, Proceedings of the 2nd International GOCE User Workshop "GOCE, The Geoid and Oceanography" (Lacoste H., ed.), ESA SP-569, 2004.

9 Hirt C, Prediction of vertical deflections from high-degree spherical harmonic synthesis and residual terrain model data, Journal of Geodesy, 84(3), (2010), 179-190, DOI 10.1007/s00190-009-0354-x.

10 Hirt C, Gruber T, Featherstone W E, Evaluation of the first GOCE static gravity field models using terrestrial gravity, vertical deflections and EGM2008 quasigeoid heights, Journal of Geodesy, 85(10), (2011), 723-740, DOI 10.1007/s00190-011-0482-y.

11 Hughes $\mathbf{C}$ W, Bingham $\mathbf{R} \mathbf{J}$, An oceanographer's guide to GOCE and the geoid, Ocean Science, 4, (2008), 15-29.

12 Janák J, Pitoňák M, Comparison and testing of GOCE global gravity models in Central Europe, Journal of Geodetic Science, 1(4), (2011), 333-347.

13 Jarvis A, Reuter H I, Nelson A, Guevara E, Hole-filled seamless SRTM data V4, 2008, available at http://srtm.csi.cgiar.org International Centre for Tropical Agriculture (CIAT).

14 Mayer-Gürr T, ITG-Grace03s: The latest GRACE gravity field solution computed in Bonn, 2007, available at http://www.igg.uni-bonn.de/apmg/ index.php?id=itg-grace03 Joint Int. GSTM and SPP Symposium, 1517 Oct. 2007, Potsdam, Germany. 
15 Mayer-Gürr T, Kurtenbac E, Eicker A, ITG-Grace2010 Gravity Field Model, 2010, available at http://www.igg.uni-bonn.de/apmg/index. php?id=itg-grace2010

16 Nagy D, Papp G, Benedek J, The gravitational potential and its derivatives for the prism, Journal of Geodesy, 74(7-8), (2000), 552-560, DOI 10.1007/s001900000116. Erratum in J. Geod. 76(8):475.

17 Newton's Bulletin: External quality evaluation reports of EGM08, 2009. Issue 4, Bureau Gravimétrique International (BGI) / International Geoid Service (IGeS).

18 Pail R, Goiginger H, Mayrhofer R, Schuh W D, Brockmann J, Krasbutter I, Höck E, Fecher T, Global gravity field model derived from orbit and gradiometry data applying the time-wise method, 2010. Presented at the ESA Living Planet Symposium 2010, Bergen, June 27 - July 2, Bergen, Norway; See also: earth.esa.int/GOCE.

19 Pail R, Bruinsma S, Migliaccio F, Förste C, Goiginger H, Schuh W D, Höck E, First GOCE gravity field models derived by three different approaches, Journal of Geodesy, 85(11), (2011), 819-843, DOI 10.1007/s00190-011-0467-x.

20 Papp G, Szücs E, Effect of the difference between surface and terrain models on gravity field related quantities, Acta Geod. Geoph. Hung., 46(4), (2011), 441-456, DOI 10.1556/AGeod.46.2011.4.6.

21 Pavlis N K, Holmes S A, Kenyon S C, Factor J K, An Earth Gravitational Model to Degree 2160: EGM2008, 2008. Presented at the 2008 General Assembly of the European Geoscience Union, Vienna, Austria, April 13-18, 2008.

22 Rummel R, Balmino G, Johannessen J, Visser P, Woodworth P, Dedicated gravity field missions - principles and aims, Journal of Geodynamics, 33, (2002), 3-20.

23 Szabó Z, Lorand Eötvös the physicist and the geological exploration, Geophysical Transactions, 45(3), (2004), 102-110. (in Hungarian).

24 Tóth G, New Combined Geoid Solution HGTUB2007 for Hungary, Observing our Changing Earth (Perugia, Italy, Winter 02/07/2007), Int Assoc. of Geod. Symposia (Sideris M G, ed.), Springer-Verlag, 2009, 405-412.

25 Tscherning C C, Rapp R H, Closed covariance expressions for gravity anomalies, geoid undulations and deflections of the vertical implied by anomaly degree variance models, Department of Geodetic Science Ohio State University, Columbus, 1974. Report 208.

26 Vergos G S, Tziavos I N, Sideris M G, On the validation of CHAMP- and GRACE-type EGMs and the construction of a combined model, Geodesy and Cartography, 55, (2006), 115-131.

27 Voigt C, Rülke A, Denker H, Ihde J, Liebsch G, Validation of GOCE products by terrestrial data sets in Germany, 2010. Geotechnologien Science Report No. 17, Observation of the System Earth from Space, Status Seminar, 04 October.

28 Zaletnyik P, Völgyesi L, Paláncz B, Modelling local GPS/levelling geoid undulations using Support Vector Machines, Periodica Polytechnica Civil Engineering, 52(1), (2008), 39-43, DOI 10.3311/pp.ci.2008-1.06. 